Muscular stimulation

\title{
A Model Study on Fascicle Selective Stimulation of Multi-Fascicular Nerves
}

\author{
Peter H. Veltink, Herman B.K. Boom and Benno K. van Veen
}

\author{
Biomedical Engineering Division \\ Department of Electrical Engineering \\ University of Twente, Enschede, The Netherlands
}

\section{ABSTRACT}

A model of nerve stimulation is presented, incorporating realistic cross-sectional geometry of a multi-fascicular nerve. The potential field within the nerve as caused by an stimulation electrode was calculated numerically. Nerve fiber excitation was modeled according to McNeal [1]. Fascicle selective stimulation was studied for electrodes placed outside the nerve, in the epineurium just outside the fascicle and inside the fascicle. The model predicts that extraneural electrodes might selectively stimulate fascicles at the surface of the nerve, while intraneural or even intrafascicular electrodes are neccessary for selective stimulation of deeper lying fascicles.

\section{INTRODUCTION}

Multi-electrode nerve stimulation configurations might be used for functional neuromuscular stimulation in order to alternately activate different parts of the same muscle and selectively stimulating different muscles. For these purposes, different electrodes should stimulate different groups of motoneurons in the nerve. In multi-fascicular nerves, fascicles may be stimulated selectively by electrodes in their neighborhood, because fascicles have different positions in the nerve [2]. Furthermore, selective stimulation of different muscles may be possible, because muscle specificity varies between fascicles [2]. In this paper a model study is presented of selective stimulation of fascicles in an example of a multi-fascicular nerve geometry.

\section{METHODS}

Computation of the electrical potential distribution

The potential distribution in a realistic geometrical nerve model was calculated numerically. The medium was assumed to be purely resistive. The nerve volume was divided into identical wedge-shaped volume elements. The nerve volume was divided in 40 layers (z-direction), each consisting of 40 rows of from 61 to 99 volume elements in $\mathrm{x}$-direction.

A dissipation functional was defined on the volume and discretized as the sum of contributions from all elements. The potentials on the discrete corner points of the volume elements was calculated by minimizing the total dissipation (variational method [3]) using iterative numerical methods.

Both the boundary condition for the model surface and the electrode itself were of the Dirichlet type (imposed potential), except for the first cross-sectional plane of the model, containing the stimulating electrode. This is a plane of symmetry and therefore a Neumann boundary condition was used here (zero current density normal to the surface).
Cross-sectional geometry of modeled nerves

As an example of a multi-fascicular human nerve, we chose the human Deep Peroneal nerve, of which a sample cross - section was obtained from Sunderland [2] (fig. 1).

\section{Electrical conductivities}

The nerve model consists of a number of compartments (fig. 1), with different conductivities. The intra-fascicular conductivity was assumed to be anisotropic ( $\sigma f x=\sigma f y=$ $0.08(\Omega \mathrm{m})-! ; \sigma f z=0.50(\Omega \mathrm{m})-!)[4]$, all other compartments were given an isotropic conductivity. The connective tissue surrounding the fascicles is rather anisotropic [1]. Therefore, we divided it into two compartments: The rather low conducting perineural sheath $(\sigma p=0.01(\Omega \mathrm{m})-!)$ enfolding conducting perineural sheath $(\sigma p=0.01(\Omega \mathrm{m})-!)$ enfolding $=0.10(\Omega \mathrm{m})-1)$, comprising the fascicles. The extraneural conductivity was assumed to be the same as the conductivity of fat tissue $(\sigma e x=0.04(\Omega \mathrm{m})-!)$ [4]. The terminating compartment of the model was assumed to have a low conductivity $(\sigma t=10-\$(\Omega \mathrm{m})-!)$.

\section{Modelling of nerve fiber excitation}

Excitation of individual nerve fibers in the nerve was modeled using the McNeal network model of myelinated nerve fiber excitation [1]. Monopolar cathodic stimulation on a single electrode was simulated. Pulse width was taken $60 \mu$ s. Pulse amplitude Va was varied. The model comprises 11 nodes of Ranvier, of which the middle one, node 0 , is nearest to the electrode. Node position L0 was defined as the distance from node 0 to the nerve cross-section through the electrode [5]. Two extreme values of $L 0$ were considered: the electrode [5]. Two extreme values of $L 0$ were to be proportional to nerve fiber diameter $\mathrm{D}[1]: \mathrm{L}=100 . \mathrm{D}$.

\section{RESULTS}

Fig. 1 shows simulated recruitment contours for both L0 $=\mathrm{L} / 2$ and $\mathrm{L} 0=0$ and for three electrode positions. The threshold stimulation amplitude TSA is defined as the stimulus pulse amplitude which depolarizes the nerve fiber up to its threshold. Ranges of TSA for each of the fascicles in the nerve and for the three electrode positions are shown in fig. 2. With an extraneural electrode only superficial in fig. 2. With (e.g. fascicle B in fig. 2a) can be stimulated with reasonable selectivity. Selective stimulation of deeper lying fascicles (e.g. fascicle A) must be expected to require intrafascicular electrodes, because the electrode is closest to the nerve fibers in the fascicle, and because of the low conducting perineurium. Some "cross-talk" to other fascicles must be expected when using an electrode lying in the epineurium just outside the fascicle (fig. $1 \mathrm{~b}$ and $2 \mathrm{~b}$ )

Fig. 2 shows that the distance between TSA contours for $\mathrm{LO}=\mathrm{L} / 2$ and $\mathrm{LO}=0$ decreases and dependency of TSA on nerve fiber diameter increases for larger distance between electrode and fascicle.

1682--IEEE ENGINEERING IN MEDICINE \& BIOLOG CH2566-8/88/0000--1682 $\$ 1.00 \odot 1988$ IEEE 


\section{DISCUSSION}

Intraneural or even intrafascicular electrodes may be expected to be necessary for selective stimulation of non-superficial fascicles of a multi-fascicular nerve. These model results must be verified experimentally and the concept of fascicle selective stimulation must be tested.

\section{REFERENCES}

[1] D R McNeal "Analysis of a Model for Excitation of Myelinated Nerve", IEEE Trans. Biomed. Eng., vol. 23, pp. 329-337, 1976.

[2] S. Sunderland, Nerves and Nerve Injuries, Edinburgh and London, Livingstone, 1968.
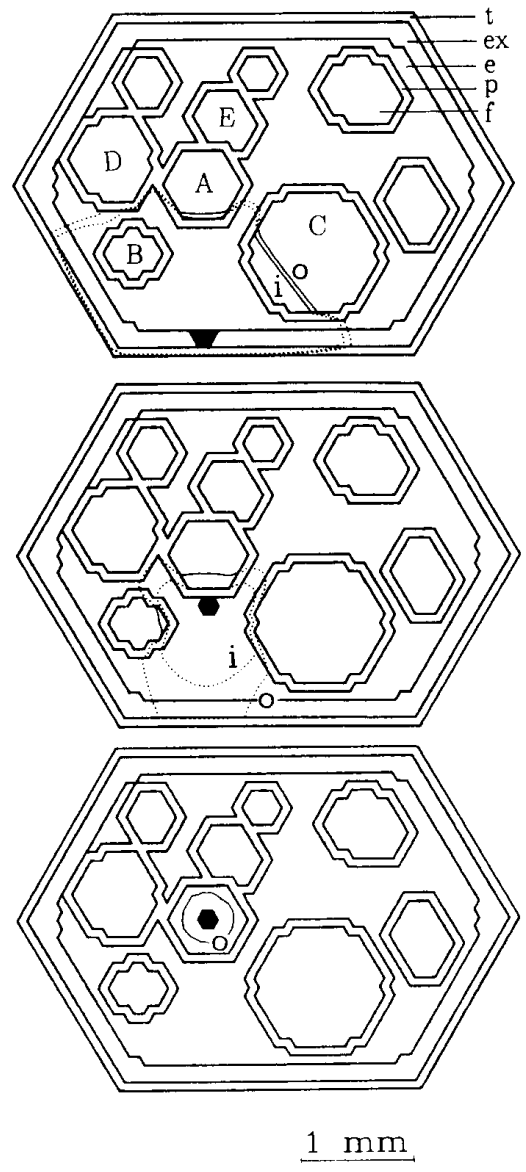
Deep Peroneal Nerve (contour i: $\mathrm{L} 0=\mathrm{L} / 2$ and contour 0 : $\mathrm{L} 0=0) . \mathrm{D}=10 \mu \mathrm{m} . \mathrm{L}=1 \mathrm{~mm}$. Electrode positions are indicated by the small filled hexagonals.

a. extraneural electrode $(\mathrm{Va}=-1.87 \mathrm{~V})$

b. epineural electrode just outside fascicle $(\mathrm{Va}=-0.59 \mathrm{~V})$

c. intrafascicular electrode $(\mathrm{Va}=-0.17 \mathrm{~V})$.

$\mathrm{b}$

c.
[3] T.C. Pilkington, M.N. Morrow and P.C. Stanley, "A Comparison of Finite Element and Integral Equation Formulations for the Calculation of Electrocardiographic Potentials", IEEE Trans. Biomed. Eng. vol. 32, 166-173, 1985

[4] L. A. Geddes and L.E. Baker, "The specific resistance of biological material - A compendium of data for the biomedical engineer and physiologist", Med. \&6 Biol. Engng., vol. 5, pp. 271-293, 1967

[6] P.H. Veltink, J.A. van Alsté and H.B.K. Boom "Simulation of Intrafascicular and Extranueral Nerve Stimulation", IEEE Trans. Biomed. Eng., vol. 35, 69-75, 1988.

Address: Peter H. Veltink, Biomedical Engineering Division, Department of Electrical Engineering, University of Twent, P.O. Box 217, $7500 \mathrm{AE}$ Enschede, the Netherlands, tel. 53-892765.

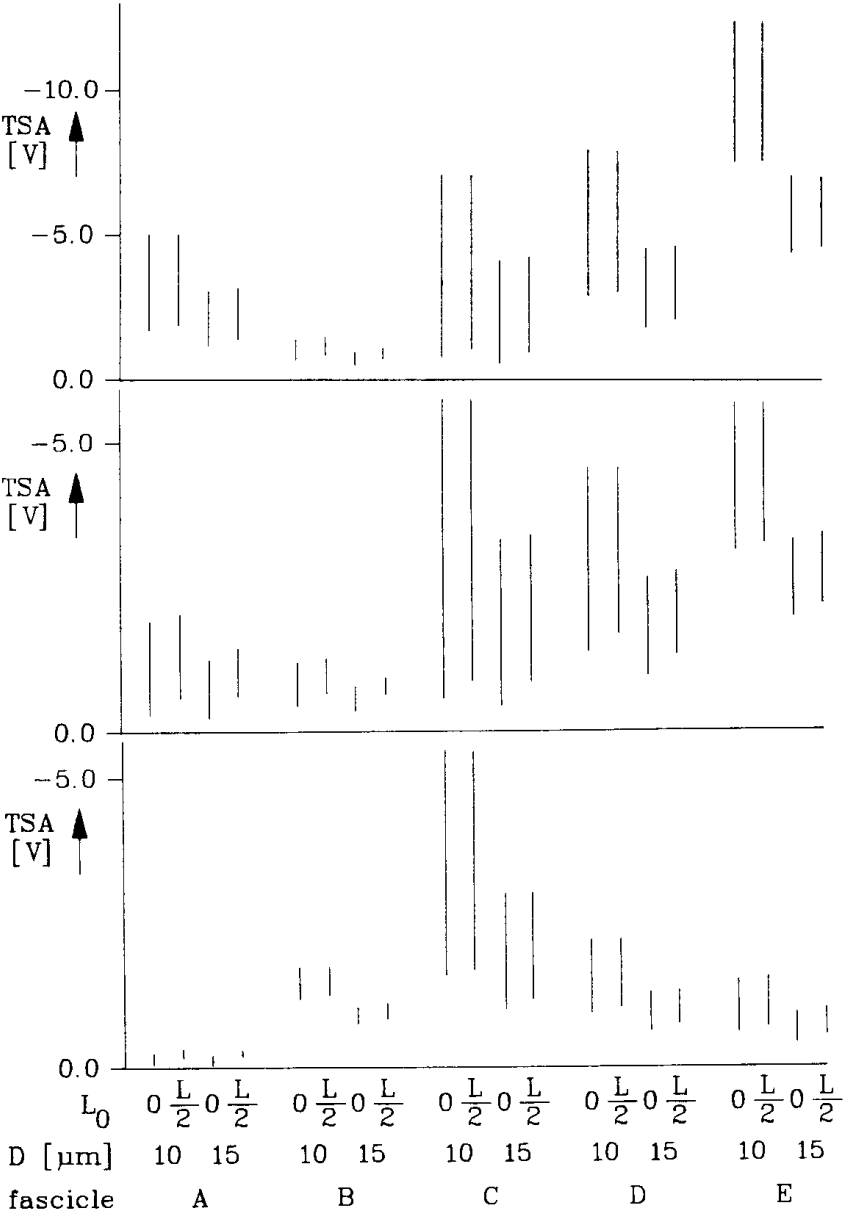

Figure 2. Variation ranges of TSA for the nerve fibers of the fascicles $\mathrm{A}-\mathrm{E}$ of the example human Deep Peroneal nerve (fig. 1). $\mathrm{D}=10 \mu \mathrm{m}$ and $\mathrm{D}=15 \mu \mathrm{m}$. $\mathrm{L} 0=0$ and $\mathrm{L} 0=\mathrm{L} / 2$. Same electrode positions as in fig. 1.

a. extraneural electrode.

b. epineural electrode just outside fascicle.

c. intrafascicular electrode. 